\title{
Hochpräzise roboterbasierte 3D-In-Prozess-Messtechnik
}

\author{
E. Csencsics, S. Ito, J. Schlarp, M. Thier, G. Schitter OVE
}

\begin{abstract}
Für effektive und adaptierbare Produktionssysteme der Zukunft stellen flexible roboterbasierte Messsysteme eine der wichtigsten Voraussetzungen dar, um die entsprechenden Anforderungen an Qualität, Produktivität und Flexibilität erfüllen zu können. Da Bewegungsunsicherheit industrieller Roboter und externe Vibrationen in Produktionsumgebungen Messungen im Mikro- bis Nanometerbereich beeinträchtigen, wird ein dual aktuierter Ansatz mit einer Messplattform, welche die Feinpositionierung des taktilen oder optischen Messsystems übernimmt, vorgeschlagen. Um Interaktionen von Messsystem und Objekt zu vermeiden, sind optische Sensoren aufgrund ihrer berührungslosen Messprinzipien sowie hohen Messraten und Auflösungen für In-Prozess-Messanwendungen besonders gut geeignet. Zur Erweiterung solcher punktweise messenden Sensoren zu echten 3D-Messsystemen werden diese mit opto-mechatronischen Kippspiegelsystemen kombiniert. Dabei wird ein integrierter Systementwurf verwendet, der alle Teilsysteme bis hin zu Ziel-Applikation von Anfang an berücksichtigt, um die Präzision und Messrate zu maximieren. Dieser Beitrag demonstriert einen solchen integrierten Ansatz am Beispiel von Kippspiegel- und Reglerentwürfen, zugeschnitten auf Raster- und Lissajous-Trajektorien, und deren Integration mit optischen Sensorsystemen.
\end{abstract}

Schlüsselwörter: In-Prozess-Messtechnik; optische Sensoren; 3D-Messtechnik; opto-mechatronische Systeme; Systemintegration

\section{High performance robot-supported 3D inline metrology.}

High precision inline measurement systems are considered one of the most important preconditions for future production, as they are a key technology to fulfill the increasing demands on quality, productivity and flexibility. To provide the required positioning uncertainty, which is typically limited by the precision of the industrial robot and external vibrations, a measurement platform for fine positioning of the tactile or optical sensing system is developed. Due to their non-contacting measurement principles, their high measurement rates and resolution, optical sensing principles are well suited for inline measurement applications. To advance single-point optical sensors to 3D measurement tools, fast steering mirrors (FSMs) are proposed to manipulate only the optical path of the sensor. This requires an integrated system design that considers all subsystems, components and the target application from the very beginning, in order to maximize system performance. In this contribution the integrated design approach is demonstrated along the FSM and controller design tailored for raster and Lissajous trajectories and their integration into a scanning optical sensor system.

Keywords: in-line metrology; optical sensors; 3D metrology; opto-mechatronic imaging systems; integrated system design

Eingegangen am 15. Mai 2018, angenommen am 12. Juli 2018, online publiziert am 1. August 2018

(c) The Author(s) 2018

\begin{abstract}
1. Einleitung
Hochpräzise In-Prozess-Messtechnik wird als eine der wichtigsten Voraussetzungen für die Produktionssysteme der Zukunft angesehen, da sie eine Schlüsseltechnologie für das Erfüllen der stetig wachsenden Anforderungen an Produktivität und Präzision darstellt $[1,2]$. Um die benötigte Flexibilität dieser In-Prozess-Messsysteme zu gewährleisten, können roboterbasierte Systeme herangezogen werden, um Positionierwege und damit das Messvolumen der Messsysteme zu erweitern $[3,4]$ und unterschiedliche Orientierungen des Messsystems, wie etwa bei der Vermessung von Freiformflächen [5], zu ermöglichen. Die Präzision industrieller Roboter ist für hochauflösende Messanwendungen im Allgemeinen allerdings nicht ausreichend und mit 30-60 $\mu \mathrm{m}$ ein bis zwei Größenordnungen über der gewünschten Messauflösung von einzelnen Mikrometern bzw. Zehntel Mikrometern [6, 7]. Allgegenwärtige Vibrationen im industriellen Produktionsumfeld, die zu einer Vibration des Messobjekts führen, sind eine weitere Störquelle für Messungen mit SubMikrometer-Auflösung [8]. Um diese Störungen zu kompensieren kann eine Messplattform verwendet werden, welche das Messsystem mit dem Roboter verbindet und die Feinpositionierung der optischen Komponenten relativ zur Oberfläche gewährleistet [8, 9].
\end{abstract}

Aufgrund kontaktfreier Messprinzipien, hoher Durchsatzraten und hoher Messauflösungen, sind, neben taktilen [10], vor allem optische Mess- und Sensorsysteme attraktiv für hochpräzise Messanwendungen direkt in der Produktionslinie [11, 12]. Um die hochpräzise Vermessung von Objekten und Oberflächen in drei Dimensionen zu ermöglichen und gleichzeitig die Leistungsfähigkeit, zufolge optischer In-Prozess-Messsysteme basierend auf StandardAutomationskomponenten, zu steigern, sind integrierte mechatronische Entwurfskonzepte erforderlich, die alle Systemkomponen-

Csencsics, Ernst, Gruppe für Advanced Mechatronic Systems, Institut für Automatisierungs- und Regelungstechnik, Technische Universität Wien, Gusshausstr. 27-29, 1040 Vienna, Austria (E-Mail: csencsics@acin.tuwien.ac.at, url: http://www.acin.tuwien.ac.at/); Ito, Shingo, Gruppe für Advanced Mechatronic Systems, Institut für Automatisierungs- und Regelungstechnik, Technische Universität Wien, Gusshausstr. 27-29, 1040 Vienna, Austria; Schlarp, Johannes, Gruppe für Advanced Mechatronic Systems, Institut für Automatisierungs- und Regelungstechnik, Technische Universität Wien, Gusshausstr. 27-29, 1040 Vienna, Austria; Thier, Markus, Gruppe fü Advanced Mechatronic Systems, Institut für Automatisierungs- und Regelungstechnik, Technische Universität Wien, Gusshausstr. 27-29, 1040 Vienna, Austria; Schitter, Georg, Gruppe für Advanced Mechatronic Systems, Institut für Automatisierungs- und Regelungstechnik, Technische Universität Wien, Gusshausstr. 27-29, 1040 Vienna, Austria 
ten bis hin zur Zielanwendung von Anfang an miteinbeziehen [13]. Die Kombination von integrierten hochperformanten mechatronischen Systemen und Bewegungssteuerung - beides grundlegende Technologien für bildgebende Systeme und Messsysteme aus dem Hightech-Segment $[14,15]$ - sowie optischer Komponenten, bildet, unter systematischer Berücksichtigung wechselseitiger Beziehungen und Einflüsse, den Ausgangspunkt für diesen Entwurfsansatz. Resultate eines solchen integrierten Entwurfsansatzes sind hochperformante bildgebende mechatronische Systeme wie Rasterkraftmikroskope (AFM) [16], konfokale Laser-Scanning-Mikroskope [17] oder Adaptive Optische Systeme [18].

Damit die typischerweise punktweise messenden optische Sensorsysteme [19] zu schnellen, flächigen Messsystemen erweitert werden können, ohne die Scanbewegung mit dem industriellen Roboters realisieren zu müssen, werden Kippspiegelsysteme (engl. fast steering mirrors, FSM) integriert, um ausschließlich den optischen Pfad des Sensorsystems zu manipulieren [20]. Zur Gewährleistung einer präzisen und effizienten Scanbewegung dieser Systeme, ist ein entsprechend integriertes System- und Reglerdesign unverzichtbar $[21,22]$

Dieser Beitrag gibt einen Überblick über die wichtigsten Konzepte und Komponenten eines roboterbasierten scannenden optischen Messsystems für In-Prozess-Messtechnik, und beschreibt den Entwurf von Subsystemen, sowie deren Implementierung.

\section{Messplattform für die In-Prozess-Messtechnik}

Relativbewegungen zwischen Messsystem und Messobjekt, z. B. aufgrund von Vibrationen oder der vergleichsweise großen Positionsunsicherheit, sowie der geringen Steifigkeit von Industrierobotern, führen zu Messfehlern oder machen hochauflösende Messungen überhaupt unmöglich. Deshalb werden hochauflösende Messungen meist in geschützten Laborumgebungen auf vibrationsisolierten Tischen oder Vorrichtungen durchgeführt, die in dieser Form in industriellen Produktionslinien nicht praktikabel sind. Um Störungen aufgrund von Umgebungsvibrationen und der Eigenschaften industrieller Roboter zu kompensieren und roboterbasierte Messsysteme mit hoher Präzision für In-Prozess-Anwendungen zu ermöglichen, kann ein neuer dual aktuierter Ansatz zur aktiven Regelung des Abstands und der Lage des Messsystems relativ zum Messobjekt in allen sechs Freiheitsgraden angewandt werden [8, 9]. Das Ziel ist es dabei hochauflösende Inspektionsgeräte, wie z. B. ein AFM, von vibrationsgeschützten Laborumgebungen direkt in einer Produktionsumgebung zur Anwendung zu bringen. In Abb. 1 ist das Systemkonzept dargestellt, welches den dual aktuierten Ansatz zeigt. Der Roboter wird für die Grobpositionierung der Messplattform eingesetzt, während diese das Messsystem trägt und dessen Feinpositionierung relativ zum Messobjekt übernimmt.

Das Ziel ist es eine künstlich steife Verbindung zwischen Objektoberfläche und Messsystem, unter der Verwendung von FeedbackRegelung und Aktuation, zu generieren, welche die hochauflösende Messung von externen Vibrationen isoliert und eine mikro- bis nanometergenaue Charakterisierung des Messobjekts direkt in der Produktionslinie ermöglicht. Gleichzeitig sorgt das Aktuationsprinzip der Messplattform für die Vibrationsentkoppelung vom Roboter. Die kompakte, starre Messplattform wird dazu über einen 6Freiheitsgrad-Aktuator, der über eine passive Gravitationskompensation zur Reduktion des Strombedarfs verfügt, mit dem Endeffektor des Roboters verbunden und bestimmt ihre Lage zum Messobjekt mit mehreren on-board Positionssensoren. Für die eigentliche Messaufgabe kann die Plattform mit einem oder mehreren taktilen oder optischen Messsystemen ausgestattet sein.

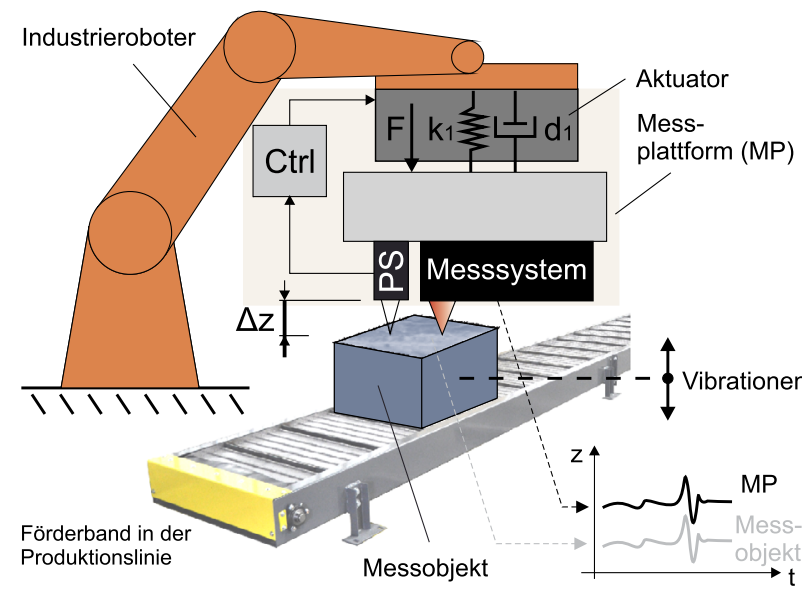

Abb. 1. Konzept einer Messplattform für Messungen im Mikro- und Nanometerbereich in vibrationsreichen Umgebungen. Die Plattform übernimmt die Feinpositionierung des Messsystems und ist über ein Interface mit einem Industrieroboter zur Grobpositionierung verbunden

2.1 In-Prozess-Messungen im Nanometer-Bereich: Aim4np Im Projekt aim4np (Automated Inline Metrology for Nanoscale Production, Website: aim4np.eu) $[8,9]$ wurde das beschriebene Konzept mit dem Ziel implementiert, ein mittels Roboter positioniertes hochauflösende AFM direkt unter Produktionsbedingungen zur Anwendung zur bringen. Abbildung 2a zeigt das speziell entwickelte hochkompakte AFM (Abmessungen: $35 \times 23 \times 40 \mathrm{~mm}^{3}$ ) mit integrierter Scannereinheit (Stellbereich: $100 \times 100 \times 20 \mu \mathrm{m}^{3}$, Nanosurf, $\mathrm{CH}$ ). Mittels Lagemessung mit 6 Positionssensoren und einer Feedback-Regelstruktur mit 6 individuell justierbaren single-inputsingle-output (SISO) Reglern kann die Plattform der Bewegung des Messobjekts mit verbleibenden Positionsunsicherheiten von $22 \mathrm{~nm}$, $17 \mathrm{~nm}$ und $14 \mathrm{~nm}$ in $\mathrm{x}-, \mathrm{y}$ - und z-Richtung folgen [23]. Dieser Ansatz verbessert die Positioniergenauigkeit, im Vergleich zu herkömmlichen Industrierobotern mit Unsicherheiten im Bereich einiger $10 \mu \mathrm{m}$ $[6,7]$, damit um drei Größenordnungen.

Im Zuge von Experimenten wurden mit dem AFM Bilder einer Teststruktur mit regelmäßigen $98 \mathrm{~nm}$ tiefen Löchern aufgenommen. Abbildung $2 \mathrm{~b}$ zeigt die Referenzmessung die unter vibrationsisolierten Laborbedingungen mit einem Standard AFM durchgeführt wurde. Im Vergleich dazu zeigt Abb. 2c das Ergebnis welches mit dem aim4np-System und dem On-board AFM in vibrationsreicher nicht-isolierter Produktionslaborumgebung (Spektrum Geschwindigkeit: max. $60 \mu \mathrm{m} / \mathrm{s}$ bei $97 \mathrm{~Hz}$, Spektrum Beschleunigung: max. $100 \mathrm{~mm} \mathrm{~s}^{2}$ bei $280 \mathrm{~Hz}$, max. Beschleunigung $2.8 \mathrm{~m} / \mathrm{s}^{2}$, max. Auslenkung $3.2 \mu \mathrm{m}$ ) aufgenommen wurde und demonstriert damit, dass nanometergenaue Vermessungen von Oberflächen außerhalb von vibrationsisolierten Präzisionslabors ermöglicht werden [23].

Das modulare Konzept (Abb. 1) ermöglicht auch die Verwendung von nicht-taktilen Messsystemen die auf der Plattform montiert werden können, sodass dieser Ansatz auch für hoch-genaue scannende optische Messsysteme für weitere Oberflächencharakterisierung und dimensionelle Messtechnik anwendbar ist.

\section{Systemstruktur Scan-basierter Messsysteme}

Unabhängig davon ob ein taktiles oder optisches Messprinzip zugrunde liegt, kann die Funktion eines Scan-basierten Messsystems in 3 Hauptaufgaben aufgeteilt werden. i) Realisierung der ScanBewegung in $\mathrm{x}$ - und $\mathrm{y}$-Richtung zur Abtastung einer Oberfläche. 
aim4np AFM

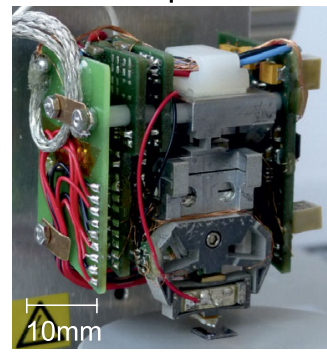

(a)

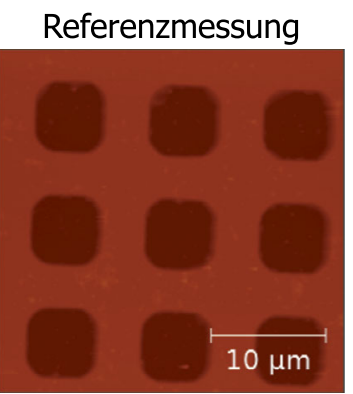

(b)

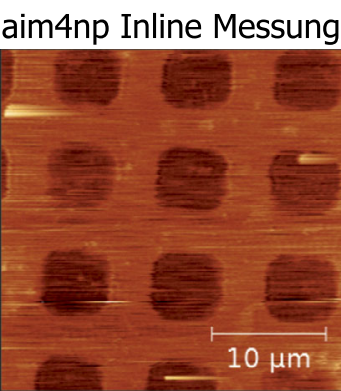

(c)

Abb. 2. AFM-Messungen eines Testgitters mit $98 \mathrm{~nm}$ tiefen Löchern. In (a) ist das kompakte AFM mit integrierter Scannereinheit und Abmessungen von $35 \times 23 \times 40 \mathrm{~mm}$ abgebildet. (b) Zeigt eine Messung mit einem Standard-AFM, aufgenommen in einer vibrationsisolierten Laborumgebung. (c) Zeigt eine Messung mit dem On-board AFM des aim4np Systems in vibrationsreicher Büroumgebung [23]

ii) Durchführung der eigentlichen Messaufgabe zur Aufnahme der Höheninformation. iii) Synchronisierte Erfassung und Verarbeitung der Mess- und Positionsdaten zur Rekonstruktion der vermessenen Oberfläche. Abb. 3 zeigt einen Überblick über die Struktur eines Scan-basierten Messsystems am Beispiel eines Laser-TriangulationSensors.

Für die Scanbewegung kann entweder das Messobjekt, wie bei manchen AFMs [24], der Messkopf selbst [25] oder, im Fall eines optischen Sensors, nur der optische Pfad, etwa mit Hilfe eine Kippspiegels (siehe roter Bereich in Abb. 3), bewegt werden [26]. Die maximale Auflösung und Messrate wird, abgesehen von den Limitierungen des eigentlichen Messsystems, maßgeblich von der Auflösung und der Bandbreite des Scanners bestimmt. Typischerweise werden Piezo- [16] oder elektromagnetische Aktuatoren [15, 26], welche die Aktuationskraft, Scanbereich und -geschwindigkeit bestimmen und Sensoren zur Positionsmessung verwendet. Die Integration der Aktoren, Sensoren und der mechanischen Struktur des Scanners ist dabei maßgeblich für die Performanz die mit einer Regelstruktur zur Positionsregelung erzielt werden kann [15]. Mögliche Führungsstrategien umfassen dabei Open-loop- [24], Closed-loop[26] und 2 Freiheitsgrad-Strukturen [27], sowie repetitive [28] und lern-basiert Ansätze [29].

Die Anforderungen der eigentlichen Messaufgabe hängen vom verwendeten Messprinzip ab. Bei taktilen Systemen, wie AFMs, wird die Bildqualität durch die Interaktion zwischen der mechanischen Messspitze und Messobjekt bestimmt und kann durch GeometrieArtefakte beeinträchtigt werden [30]. Bei optischen Prinzipien sind der Beleuchtungspfad (Umgebungslicht, Staub, etc.) und die Interaktion zwischen Laser-/Lichtstrahl und dem Messobjekt maßgeblich, da die Intensität des zurück gestreuten Lichts maßgeblich für das Signal-zu-Rausch-Verhältnis des Sensors ist [31]. Weiters sind der Durchmesser des Messflecks und die Messrate wichtige Faktoren für die resultierende Auflösung des Gesamtsystems [12].

Eine präzise synchronisierte Datenakquisition ist daher erforderlich um die Daten des Messsystems mit den Positionsdaten des Scanners korrekt verknüpfen zu können. Latenzzeiten durch die Datenverarbeitung im optischen Sensor müssen für das Datenakquisitionssystem berücksichtigt werden, um synchronisierte Datenvektoren zu garantieren. Sollte z. B. ein konstanter zeitlicher Versatz zwischen den Datenvektoren vorliegen, würden sich bei einem Raster-Scan unscharfe Objektkanten in der schnellen Scan-Achse ergeben [32]. Das Datenakquisitionssystem muss daher alle Latenzen im System systematisch berücksichtigen, die darüber hinaus auch deterministisch sein müssen. Zur Rekonstruktion der vermessenen Oberfläche

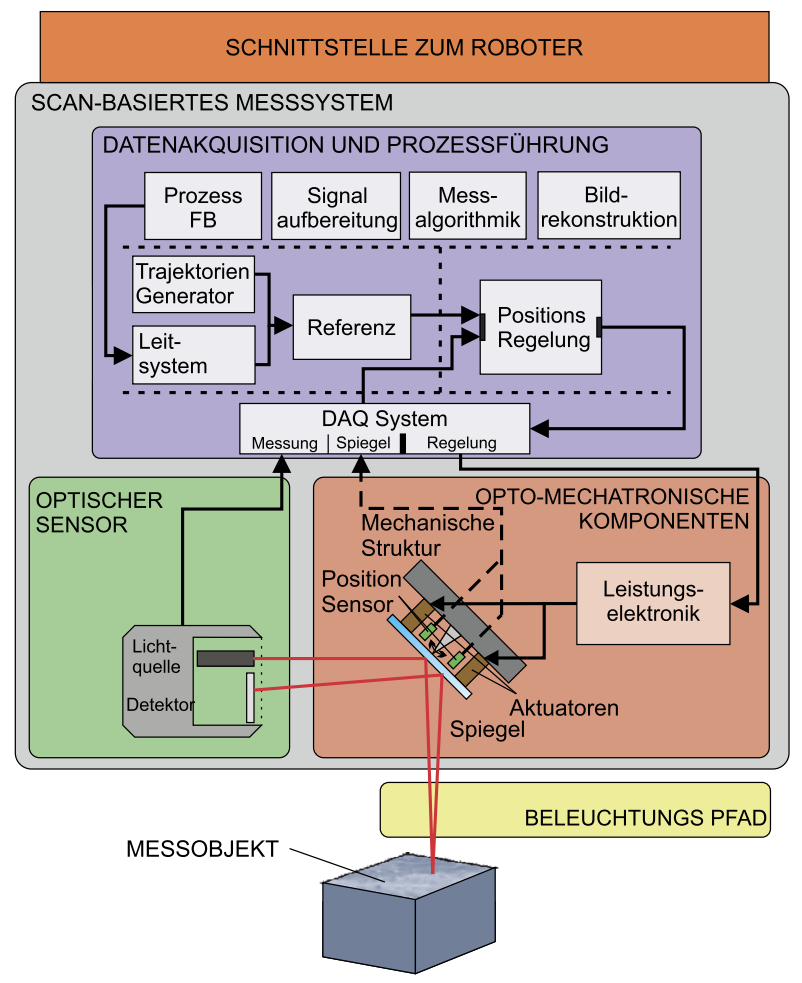

Abb. 3. Hochintegriertes Scan-basiertes optisches Messsystems für die dreidimensionale Vermessung von Messobjekten. Das scannende optische Messsystem bestehend aus dem optischem Sensor, dem opto-mechatronischen Scanner, dem Datenakquisitionssystem und der Regelung, sowie der Softwarebausteine zur Prozessführung und Datenauswertung

sind Funktionen zur Aufbereitung der Messsignale, sowie entsprechende Mess- und Korrekturalgorithmen zur Kompensation systematischer Fehler (z. B. Scanner Bow, Plane Tilt) erforderlich (siehe Abb. 3). Die Prozessführung generiert die entsprechenden ScanTrajektorien und adaptiert diese automatisch für die entsprechende Anwendung [33].

\section{Design Scan-basierter opto-mechatronischer Sensorsysteme}

Die Entwicklung von Methoden zum systematischen Entwurf von integrierten Scan-basierten optischen Sensoren und deren opto- 

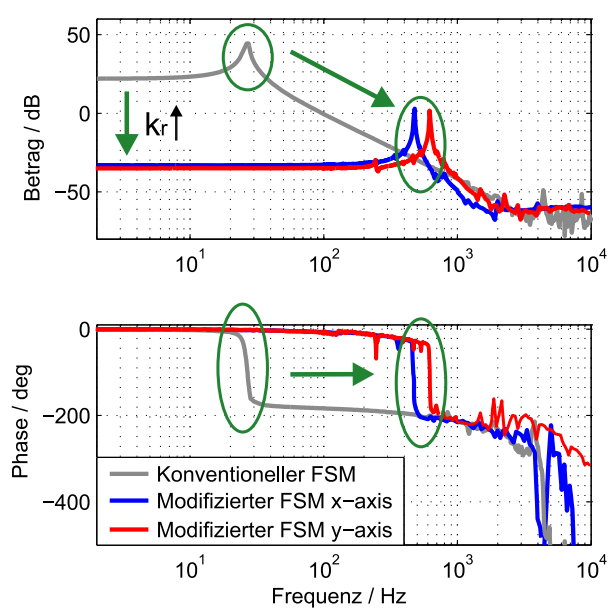

(a)

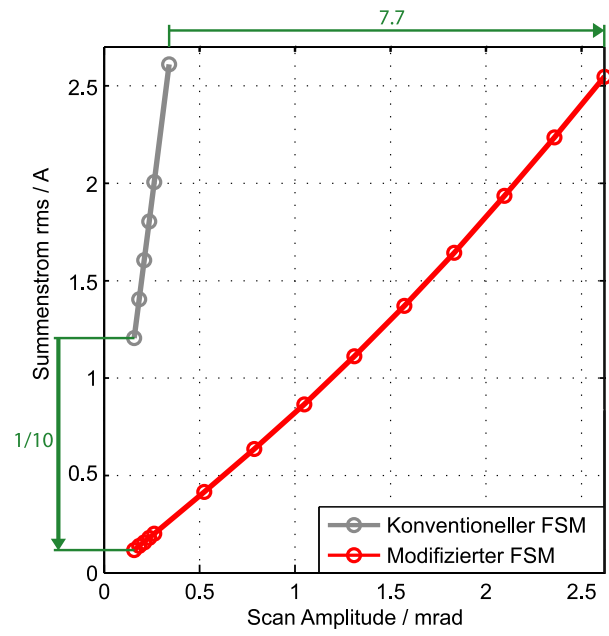

(b)

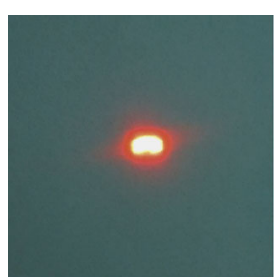

(c)

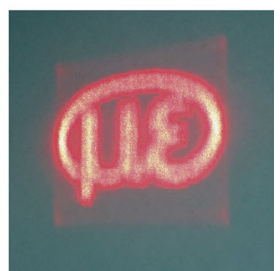

(d)

Abb. 4. Effizienzsteigerung durch Systemintegration. (a) Zeigt die Abstimmung der Systemresonanzen auf die Scanfrequenzen und (b) den benötigen Strom in Abhängigkeit der Scanamplitude für das konventionelle (grau) und das modifizierte (rot) FSM-System. Die Projektion eines Projektionssystems mit dem konventionellen bzw. modifizierten FSM bei maximaler Scanamplitude ist in (c) bzw. (d) abgebildet (Farbabbildung online.)

mechatronischen Komponenten wird aktuell im Christian Doppler Labor für Präzisionstechnologie für Automatisierte In-Line Messtechnik vorangetrieben, um die Grundlage für voll-integrierte präziser 3D-Messsysteme der nächsten Generation zu schaffen.

\subsection{Systemgeometrie}

Zur Erweiterung punkt- oder linienweise messender optischer Sensoren [19] zu echten 3D-Messsystemen, werden optische Sensorsysteme mit opto-mechatronischen Kippspiegelsystemen integriert. Die Anordnung, Spiegelgröße und Stellbereich des Scanners, sowie das optische Pfaddesign, werden dabei maßgeblich vom verwendeten Sensorprinzip bestimmt. Die Systemgeometrie bestimmt darüber hinaus die Algorithmen zur Rekonstruktion der vermessenen Oberfläche aus den Mess- und Positionsdaten. Abbildung 3 zeigt beispielhaft die Geometrie eines scannenden Laser-TriangulationSensor Systems [31], welches den hin- und rücklaufenden Laserpfad mittels eines Kippspiegels manipuliert [20]. Dafür wird eine größere Spiegelapertur benötigt, da beide Laserpfade über den Spiegel geführt werden müssen, was sich nachteilig auf das Trägheitsmoment und damit auf die Scangeschwindigkeit auswirkt. Andererseits reduziert diese Anordnung systematische Fehler und macht den gesamten Messbereich des Sensors zur Vermessung des Objekts nutzbar.

Bei der Integration mit konfokal chromatische Sensoren [12] ist ebenfalls eine größere Spiegelapertur erforderlich, da der gesamte Weißlichtkegel des Sensorkopfs über den Spiegel geführt werden muss [32]. Die Spiegelgröße kann dabei durch Variation des Abstandes von Scanner zu Sensor gegen lateralen Scanbereich abgewogen werden.

Darüber hinaus sind, abhängig vom optischen Sensorprinzip, auch andere Sensorgeometrien denkbar, die z. B. die Verwendung kleinerer Spiegel ermöglichen, aber einen geringeren Messbereich aufweisen [20].

\subsection{Scan Trajektorien}

Die am weitest verbreitete Trajektorie zum Scannen einer Flächen ist die Raster-Trajektorie, die in unterschiedlichen wissenschaftlichen Instrumenten, wie AFMs [24] und Fertigungsprozessen angewandt wird. Sie wird durch ein schnelles und eine langsames Dreieckssignal an den beiden Scanachsen generiert und bietet eine einheitliche Auflösung über den gesamten Scanbereich, was eine konstante Pixel-Clock für die Zuweisung von Datenpunkten zu einem Referenzraster bedeutet. Obwohl die Raster-Trajektorie der Standard für viele wissenschaftliche Applikationen ist, stellt sie oft auch die Limite für die erreichbare Scangeschwindigkeit dar [34]. Für ein DreiecksReferenz-Signal müssen mindestens die ersten 7-11 Harmonischen der Grundwelle innerhalb der Systembandbreite liegen, sodass eine hohe Reglerbandbreite benötigt wird, die wiederum hohe Anforderungen an die mechanische Struktur zur Folge hat [15].

Die Lissajous-Trajektorie stellt eine Alternative zu Raster-Trajektorien dar und wird ebenfalls zunehmend in Präzisionsanwendungen verwendet [34]. Sie wird durch Sinussignale mit definierten Frequenzen an beiden Systemachsen generiert, wobei die Frequenzen Auflösung und Bildrate definieren [35]. Die Auflösung ist in der Mitte des Scanbereichs am geringsten und nimmt zu den Rändern hinzu. Als $\mathrm{MaB}$ für die minimale Auflösung kann der maximale Abstand zwischen zwei Kreuzungen der Trajektorie mit den Hauptachsen definiert werden. Die Auflösung baut sich schrittweise mit fortschreitender Dauer des Scans auf, sodass bereits nach einem Bruchteil der Scandauer ein Überblick über den gesamten Scanbereich zur Verfügung steht. Diese Multi-Resolution-Eigenschaft [35] kann für variable schnelle Messsysteme mit hohem Durchsatz ein entscheidender Vorteil sein.

\subsection{Integriertes Kippspiegel-Design}

Mit der erforderlichen Spiegelapertur abgeleitet aus der Systemgeometrie kann der Scanner entworfen werden. Typischerweise werden Lorentz-aktuierte FSM-Systeme verwendet, da diese über einen gröBeren Stellbereich von bis $z u \pm 1.5^{\circ}$ verfügen und Bandbreiten von etwa $800 \mathrm{~Hz}$ haben (Type: OIM101, Optics In Motion LLC, Long Beach, USA) [26]. Die Aufhängungen dieser Systeme haben geringe Steifigkeit in den aktuierten rotatorischen Freiheitsgraden und hohe Steifigkeiten in den nicht-aktuierten Freiheitsgraden. Aufgrund der geringen Steifigkeit wird die Systemdynamik hauptsächlich durch das Trägheitsmoment des Spiegels und seines Trägers bestimmt, so- 


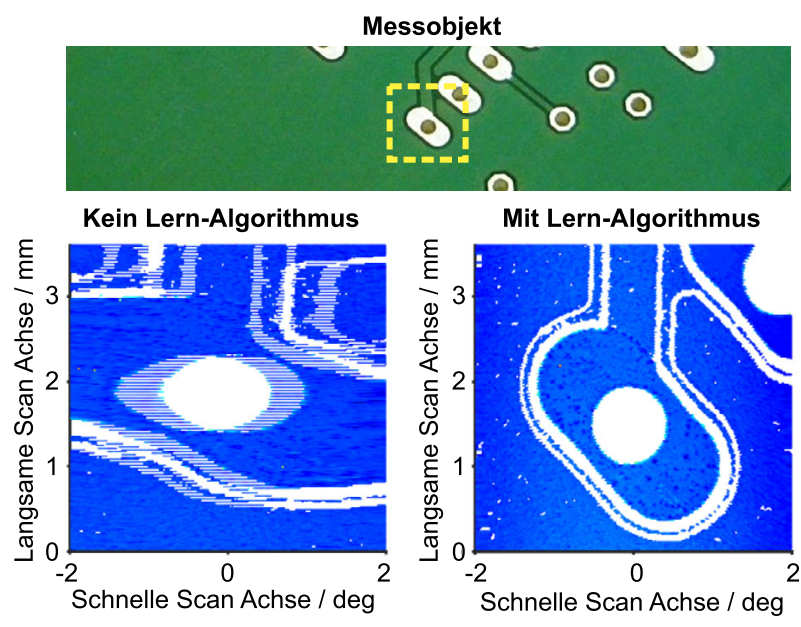

Abb. 5. Modellfreier iterativer Lern-Algorithmus kompensiert Nichtlinearitäten und die Dynamik des Scanners anhand vorhergehender Scanfehler und ermöglicht eine deutliche Verbesserung des Messergebnisses (Farbabbildung online.)

dass ein Spiegelträger mit möglichst geringem Trägheitsmoment angestrebt wird. Des Weiteren werden durch das Systemdesign strukturelle Moden der mechanischen Struktur zu möglichst hohen Frequenzen verschoben und eine gute Entkopplung der beiden aktuierten Freiheitsgrade sichergestellt, um keine Aktuatorleistung zur Unterdrückung des Übersprechens zu benötigen [26]

Interne optische Sensoren (OIM101, Optics In Motion LLC) werden zur Messung und Regelung der Spiegelposition verwendet.

Im Sinne eines integrierten Systemdesigns sollte das Kippspiegelsystem bereits an die entsprechende Ziel-Trajektorie angepasst sein. Für Raster-Trajektorien, mit ihrem abnehmenden aber breiten Amplitudenspektrum, sind diese wenig steifen Systeme in Kombination mit beispielsweise PID Reglern [22] gut geeignet. Liegen die Frequenzen der Referenz-Signale innerhalb der Systembandbreite, sind diese Systeme auch für Lissajous-Trajektorien geeignet. Zusammen mit auf die Trajektorie abgestimmten Reglern (siehe Abschn. 4.4) wird, um die gleich Auflösung wie mit einer Raster-Trajektorie zu erhalten, aufgrund der höheren fundamentalen Frequenzen der Referenzsignale, in diesem Fall allerdings der doppelte Aktorstrom benötigt [21]. Das limitiert die maximale Scanamplitude bei höheren Auflösungen und damit den Messbereich. Wird der Scanner jedoch auf die gewünschte Lissajous-Trajektorie abgestimmt, indem die Resonanz-Frequenzen durch Versteifung der Achsen zu den Referenzfrequenzen verschoben werden, kann der Stromverbrauch für die gleiche Trajektorie deutlich reduziert werden [26]. In Abb. 4a sind die Systemdynamiken eines konventionellen und eines modifizierten Kippspiegelsystems dargestellt, bei dem die Systemresonanzen beider Achsen, durch individuelle Erhöhung der Steifigkeit, zu den Frequenzen des jeweiligen Referenzsignals verschoben sind. Die höhere Verstärkung der Strecke an diesen Frequenzen ermöglicht eine Reduktion des Stromverbrauchs um 90 \% im Vergleich zum konventionellen System, was in Abb.4b dargestellt ist. Gleichzeitig ergibt sich bei gleicher maximaler Stromaufnahme eine Vergrößerung des Scanbereichs um einen Faktor 60 [26]. Abbildung 4c und $\mathrm{d}$ demonstrieren diese Vergrößerung des Scanbereichs anhand einer Laserprojektion.

\subsection{Trajektorienabhängige Regelstruktur}

Wie im Fall des Scanners muss auch die Regelstruktur auf die ZielTrajektorie zugeschnitten werden, um die maximale Leistungsfä- higkeit zu erhalten. Ausgehend von einer klassischen FeedbackRegelstruktur entsprechen etwa PID Regler den spektralen Anforderungen von Raster-Trajektorien [22]. Um den Regelfehler weiter zu reduzieren, kann zusätzlich eine Vorsteuerung ergänzt werden, die aber typischerweise sehr genau parametrierte Modelle der Systemdynamik benötigen. Für periodische Raster-Trajektorien kann alternative auch frequenzselektive modellfreie Iterative Learning Control (ILC) eingesetzt werden, welche aus Regelfehlern vorhergehender Zyklen lernt und das Referenzsignal dementsprechend anpasst [29]. Abbildung 5 zeigt das Messergebnis einer Leiterplatte, vermessen mit einem scannenden 3D-Konfokalsensorsystem mit Feedback geregeltem Kippspiegel [32], der einer Raster-Trajektorie ohne und mit aktivem Lern-Algorithmus folgt. Da der Lern-Algorithmus Nichtlinearitäten und die Dynamik des Scanners ausschließlich bei der Grundfrequenz und den Harmonischen der Ziel-Trajektorie lernt, kann der Regelfehler um 99,8 \% im Vergleich zum reinen FeedbackFall reduziert werden.

SISO-Regler für Lissajous-Trajektorien können aufgrund des schmalbandigen Spektrums der Sinus-Referenz auf die jeweilige Achse zugeschnitten werden und weisen eine lokalisierte hohe Reglerverstärkung nur an der entsprechenden Frequenz auf. Um auch das Übersprechen zwischen den Scanachsen zu unterdrücken, werden Dual-Tone (DT) Regler verwendet, die an den ReferenzFrequenzen beider Achsen eine hohe Reglerverstärkung aufweisen [21]. Beim Folgen von Lissajous-Trajektorien kann mit diesem zugeschnittenen Regler im Vergleich zu einem klassischen PID Regler der Regelfehler um eine Größenordnung, auf unter 0,8 \% der Scanamplitude, reduziert werden.

\subsection{Datenakquisition und Bildrekonstruktion}

Entsprechend dem Konzept in Abb. 3 wurde der Prototyp eines scannenden 3D-Laser-Triangulation-Sensors (Type: ILD 2300-100, Micro-Epsilon $\mathrm{GmbH}$, Germany) mit einem Kippspiegelsystem (Type: OIM102, Optics In Motion LLC, Long Beach, USA) entwickelt [25]. Die Datenakquisition, zur synchronen Erfassung der Messdaten und der Spiegelposition, wurde auf einem echtzeitfähigen RapidPrototyping System (dSPACE Type: DS1202, dSPACE GmbH, Germany) mit einer Samplingrate von 20 kHz, 3 Eingängen (Positionssignale beider Spiegelachsen und Sensorausgang) und 2 Ausgängen (Referenzsignale für Spiegelachsen) implementiert.

Mit der Systemgeometrie kann über geometrische Abbildungsvorschriften aus den beiden Datenvektoren die Oberfläche des gemessenen Objekts rekonstruiert und dabei systematische Fehler, wie Scanner Bow, kompensiert werden [25]. Um Verkippungen der Objektebene zu korrigieren, wird ein RANSAC Algorithmus verwendet, der die verkippte Ebene schätzt und korrigiert. Abbildung $6 a$ zeigt den Ausschnitt einer Leiterplatte, welche mit dem ersten Prototyp des scannenden Triangulations-Sensors vermessen wurde. Das Messergebnis in Abb. 6b zeigt den IC im Messbereich, sowie die umgebenden SMD Bauteile mit einer vertikalen bzw. lateralen Auflösung von $15 \mu \mathrm{m}$ bzw. $580 \mu \mathrm{m}$ und einer Messrate von 1 Frame/s. Um die Flexibilität des Systems applikationsabhängig zu erhöhen und eine automatische Erkennung relevanter Objektmerkmale zu ermöglichen, kann das System um entsprechende Clustering-Algorithmen und eine entsprechende Prozess-Führung ergänzt werden [33]. So können beispielsweise Bereiche mit relevanten Objektmerkmalen im Messfeld erkannt werden, sodass das System diese, mit neu konfigurierten Trajektorien mit entsprechendem Offset und Amplitude, automatisch mit höherer Auflösung vermessen kann.

In Summe konnte gezeigt werden, dass durch die Integration von optischen Sensoren, Mechatronik und Regelungstechnik intelligente und flexible optische 3D-Messsysteme entworfen und implementiert werden können. 


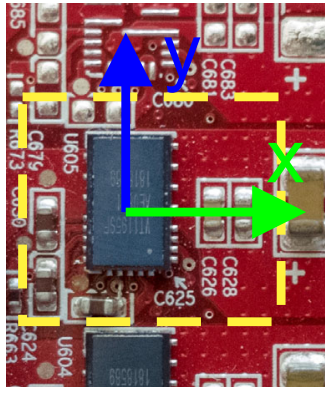

(a)

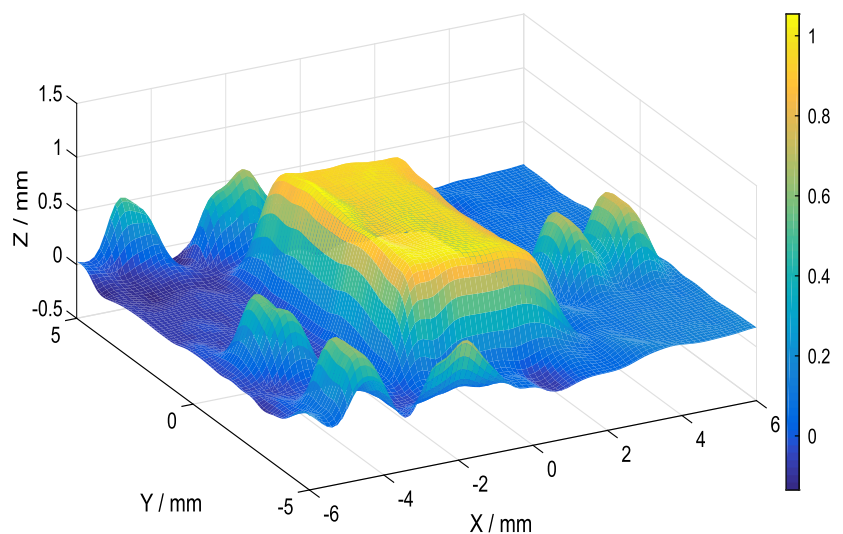

(b)

Abb. 6. Messergebnis eines Leiterplattenbereichs mit einem 3D-Triangulations-Sensorsystem. (a) Zeigt den Scanbereich (gelb) und (b) die Oberfläche, welche aus den aufgenommenen Vektoren der Mess- und Positionsdaten rekonstruiert wird

\section{Zusammenfassung und Schlussfolgerung}

Dieser Beitrag beschreibt grundlegende Konzepte flexibler roboterbasierter In-Prozess-Messsysteme mit einem genaueren Blick auf den Entwurf scannender optischer Sensorsysteme. Um roboterbasierte Messungen im Mikro- bis Nanometerbereich direkt in der Produktionslinie zu ermöglichen, wurde ein dual aktuierter Ansatz präsentiert, bei dem die große Positionsunsicherheit von Industrierobotern (Grobpositionierung) und der Einfluss externer Vibrationen mittels einer Messplattform (Feinpositionierung), die das Messsystem trägt und aktiv der Bewegung des Messobjekts nachgeführt wird, kompensiert werden. Zur Erweiterung von optischen punkt- und linienweise messenden Sensorsystemen zu flächigen 3D-Messsystemen, werden diese mit abgestimmten opto-mechatronischen Kippspiegelsystemen kombiniert. Die Berücksichtigung der Zielapplikation, sowie darauf abgestimmter Trajektorien, von Beginn des Systementwurfprozesses an ist dabei notwendig, um die Leistungsfähigkeit des Gesamtsystems zu maximieren. Dieser Ansatz wurde anhand des Entwurfs von Kippspiegelsystemen und deren Regelstruktur für Raster- und Lissajous-Trajektorien demonstriert und die damit verbundene Leistungssteigerung hinsichtlich Präzision und Energieverbrauch aufgezeigt.

\section{Danksagung}

Open access funding provided by TU Wien (TUW). Wir bedanken uns für die finanzielle Unterstützung durch das Bundesministerium für Digitalisierung und Wirtschaftsstandort und die Nationalstiftung für Forschung, Technologie und Entwicklung sowie durch die MICRO-EPSILON MESSTECHNIK GmbH \& Co. KG und ATENSOR Engineering and Technology Systems $\mathrm{GmbH}$.

Open Access This article is distributed under the terms of the Creative Commons Attribution 4.0 International License (http://creativecommons.org/ licenses/by/4.0/), which permits unrestricted use, distribution, and reproduction in any medium, provided you give appropriate credit to the original author(s) and the source, provide a link to the Creative Commons license, and indicate if changes were made.

\section{Literatur}

1. Imkamp, D., Schmitt, R., Berthold, J. (2012): Blick in die Zukunft der Fertigungsmesstechnik - Die VDI/VDE-GMA Roadmap Fertigungsmesstechnik 2020. Techn. Mes., 10(79).
2. Rahlves, M., Seewig, J. (2009): Optisches Messen technischer Oberflächen: Messprinzipien und Begriffe. Berlin: Beuth Verlag $\mathrm{GmbH}$.

3. Changwei, L., Bangming, L., Sijiong, Z. (2014): Phase retrieval using a modified shack hartmann wavefront sensor with defocus. Appl. Opt., 53(4), 618-624.

4. Rejc, J., Cinkelj, J., Munih, M. (2009): Dimensional measurements of a gray-iron object using a robot and a laser displacement sensor. Robot. Comput.-Integr. Manuf., 25(1), 155-167.

5. Iglesias, I., Sebastian, M., Ares, J. (2015): Overview of the state of robotic machining: current situation and future potential. Proc. Eng., 152, 911-917.

6. ABB Asea Brown Boveri Ltd (2017, July): IRB 40 industrieroboter. Product Website. https://library.e.abb.com.

7. Kuka Roboter Schweiz AG (2017, July): KR quantec extra. Product website: https:// www.kuka.com/en-ch/products/robotics-systems/industrial-robots/kr-quantec-extra.

8. Thier, M., Saathof, R., Hainisch, R., Schitter, G. (2015): Vibration compensation platform for robot-based nanoscale measurements. In 15th international conference of the EUSPEN.

9. Thier, M., Saathof, R., Csencsics, E., Hainisch, R., Sinn, A., Schitter, G. (2015): Design and control of a positioning system for robot-based nanometrology. Automatisierungstechnik, 63(9), 727-738.

10. Savio, E., De Chiffre, L., Schmitt, R. (2007): Metrology of freeform shaped parts. CIRP Ann., 56(2), 810-835.

11. Vision, F.-A. (2009): Marktstudie 3-D-Messtechnik in der deutschen Automobil- und Zulieferindustrie. Stuttgart: Fraunhofer Verlag.

12. Harding, K. (2013): Handbook of optical dimensional metrology. London: Taylor \& Francis.

13. Hyungsuck, C. (2003): Opto-mechtronic systems handbook. Boca Raton: CRC Press LLC.

14. Kluk, D. J., Boulet, M. T., Trumper, D. L. (2012): A high-bandwidth, high-precision, two-axis steering mirror with moving iron actuator. Mechatronics, 22(3), 257-270.

15. Munnig Schmidt, R., Schitter, G., Rankers, A., van Eijk, J. (2014): The design of high performance mechatronics. 2. ed. Warsaw: Delft University Press.

16. Schitter, G., Astrom, K. J., DeMartini, B. E., Thurner, P. J., Turner, K. L., Hansma, P. K. (2007): Design and modeling of a high-speed afm-scanner. IEEE Trans. Control Syst. Technol., 15(5), 906-915

17. Yoo, H., van Royen, M. E., van Cappellen, W. A., Houtsmuller, A. B., Verhaegen, M., Schitter, G. (2014): Automated spherical aberration correction in scanning confocal microscopy. Rev. Sci. Instrum., 85, 123706.

18. Kentischer, T. J., Bernert, A., Gantzert, L., Schelenz, T., Schmidt, W. (1996): A fast tracking mirror for adaptive optics. Astrophys. Space Sci., 239(2), 213-219.

19. Salzberger, J. (2013): In "Optisch, berührungslos, präzise - optische Messtechnik für die Qualitätssicherung im Prozess," Laser + Photonik (Vol. 4, S. 50-53).

20. Schlarp, J., Csencsics, E., Schitter, G. (2018): Optical scanning of laser line sensors for 3 dimaging. Appl. Opt., 57(18), 5242-5248.

21. Csencsics, E., Saathof, R., Schitter, G. (2016): Design of a dual-tone controller for lissajous-based scanning of fast steering mirrors. In 2016 American control conference, Boston, MA, USA.

22. Csencsics, E., Schitter, G. (2017): Parametric pid controller tuning for a fast steering mirror. In 1st IEEE conference on control technology and applications, Kohala Coast, Hawaii, USA. 
23. Thier, M., Hainisch, R., Schitter, G., Saathof, R. (2016): Metrology platform to enable in-line nanometrology. In Industrial technologies, Amsterdam, The Netherlands.

24. Schitter, G., Thurner, P. J., Hansma, P. K. (2008): Design and input-shaping control of a novel scanner for high-speed atomic force microscopy. Mechatronics, 18(5), 282288

25. Schlarp, J., Csencsics, E., Schitter, G. (2018): Optical scanning of a laser sensor for $3 \mathrm{~d}$ imaging. In IEEE transactions on instrumentation and measurement, submitted for publication.

26. Csencsics, E., Schitter, G. (2016): System design and control of a resonant fast steering mirror for lissajous-based scanning. IEEE/ASME Trans. Mechatron., 22(5), 1963 1972.

27. Schitter, G., Stemmer, A., Allgöwer, F. (2004): Robust two-degree-of-freedom control of an atomic force microscope. Asian J. Control, 6(2), 156-163.

28. Steinbuch, M., Weiland, S., Singh, T. (2007): Design of noise and period-time robust high-order repetitive contrtol with application to optical storage. Automatica, 43(12), 2086-2095

\section{Autoren}

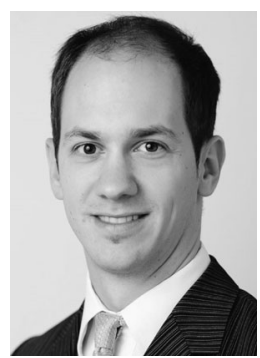

\section{Ernst Csencsics}

ist Postdoctoral Researcher am Institut für Automatisierungs- und Regelungstechnik (ACIN) der TU Wien, Österreich. Er absolvierte sein Master- (2014) und Doktoratsstudium (2017) im Fachbereich Elektrotechnik an der TU Wien. In seiner Forschung beschäftigt er sich mit dem integrierten Design und der Regelung hochperformanter mechatronischer Systeme, der Integration optischer und mechatronischer Komponenten und In-Prozess-Messsystemen.

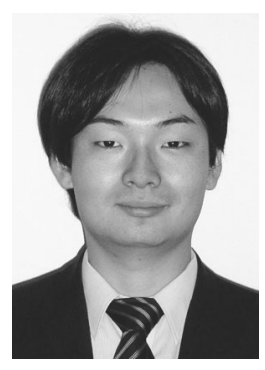

\section{Shingo Ito}

absolvierte sein Masterstudium im Fachbereich Mechanik und Industrietechnik an der University of Toronto, Kanada (2007) und sein Doktoratsstudium im Fachbereich Elektrotechnik an der TU Wien (2015). Von 2007 bis 2010 arbeitete er als Ingenieur für Regelungstechnik bei der Yaskawa Electric Corporation, Kitakyushu, Japan. Aktuell ist er als Postdoctoral Researcher am Institut für Automatisierungs- und Regelungstechnik (ACIN) der TU Wien tätig. Seine Forschungsinteressen umfassen den Entwurf und die Regelung hochpräziser mechatronischer Systeme für Produktion, Inspektion und Automation.

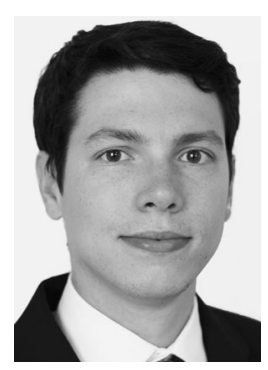

\section{Johannes Schlarp}

absolvierte sein Masterstudium im Fachbereich Elektrotechnik and der TU Wien (2017) und ist aktuell als Doktorand am Institut für Automatisierungs- und Regelungstechnik (ACIN) der TU Wien tätig. In seiner Dissertation beschäftigt er sich mit hochpräzisen mechatronischen Systemen für automatisierte In-Prozess-Messtechnik.
29. Ito, S., Yoo, H. W., Schitter, G. (2017): Comparison of modeling-free learning control algorithms for galvanometer scanner's periodic motion. In 2017 IEEE international conference on advanced intelligent mechatronics.

30. Shen, J., Zhang, D., Zhang, F., Gan, Y. (2017): Afm tip-sample convolution effects for cylinder protrusions. Appl. Surf. Sci., 422, 482-491.

31. Donges, A., Noll, R. (2015): Laser measurement technology. Atlanta: Springer

32. Ito, S., Poik, M., Csencsics, E., Schlarp, J., Schitter, G. (2018): Scanning chromatic confocal sensor for fast 3d surface characterization. In ASPE/euspenSummer topical meeting on advancing precision in additive manufacturing. submitted for publication.

33. Schlarp, J. Csencsics, E, Schitter, G. (2018): Feature detection and scan area selection for 3d laser scanning sensors. In 2018 IEEE/ASME international conference on advanced intelligent mechatronics (AIM).

34. Tuma, T., Lygeros, J., Kartik, V., Sebastian, A., Pantazi, A. (2012): High-speed multiresolution scanning probe microscopy based on lissajous scan trajectories. Nanotechnology, 23(18), 185501

35. Tuma, T., Lygeros, J., Sebastian, A., Pantazi, A. (2012): Optimal scan trajectories for high-speed scanning probe microscopy. In 2012 American control conference.

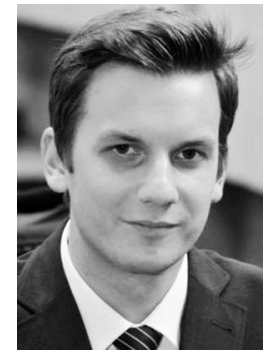

\section{Markus Thier}

absolvierte sein Masterstudium im Fachbereich Elektrotechnik an der TU Wien (2012) und war von 2012 bis 2017 als Doktorand am Institut für Automatisierungs- und Regelungstechnik (ACIN) der TU Wien tätig. Seine Forschung beschäftigte sich mit Konzepten für Vibrationsisolation für In-ProzessMesssysteme, mechatronischem Systemdesign und Wellenfrontsensorik. Aktuell arbeitet er bei Magna Powertrain im Bereich Systemintegration elektrischer Antriebssysteme.

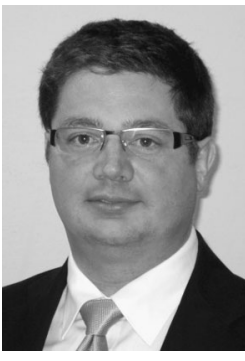

\section{Georg Schitter}

ist Professor für Advanced Mechatronic Systems am Institut für Automatisierungs- und Regelungstechnik (ACIN) der TU Wien. Er absolvierte das Studium Elektrotechnik an der TU Graz, Österreich (2000) sowie ein Diplom in Informationstechnik und das Doktoratsstudium an der ETH Zürich, Schweiz (2004). Nach mehrjährigen Forschungsaufenthalten an der UCSB, USA, und TU Delft, Niederlande wurde er 2010 an die TU Wien berufen. Seine primären Forschungsinteressen befassen sich mit der Entwicklung integrierter Systemlösungen mechatronischer Systeme, inklusive neuer systemund regelungstechnischer Methoden, vor allem für Anwendungen in der Hochtechnologie, wissenschaftlicher Instrumentierung und bildgebende mechatronische Systeme, wie AFM, scanning Laser und LIDAR-Systeme, Teleskopsysteme, Adaptive Optik, und Lithographiesysteme für die Halbleiterindustrie. Für seine Forschung wurden Schitter einige Stipendien und Preise zugesprochen, darunter Stipendien des FWF und der Österreichischen Akademie der Wissenschaften sowie Best-Paper-Awards des IFAC Journal Mechatronics und des Asian Journal of Control. Schitter war Associate Editor der IFAC Journals Control Engineering Practice und Mechatronics sowie des Conference Editorial Boards der IEEE Control Systems Society (CSS) und der IEEE Transactions on Mechatronics. 